\title{
Monolayer to MTS: using SEM, HIM, TEM and SERS to compare morphology, nanosensor uptake and redox potential in MCF7 cells
}

\author{
L. E. Jamieson ${ }^{\text {a }}$, A. P. Bell ${ }^{\mathrm{b}}$, D. J. Harrison ${ }^{\mathrm{c}}$, C. J. Campbel1*a \\ ${ }^{a}$ EaStCHEM, School of Chemistry, University of Edinburgh, Edinburgh, EH9 3JJ, UK. E- \\ mail: colin.campbell@ed.ac.uk; ${ }^{b}$ Advanced Microscopy Laboratory, CRANN, Trinity College \\ Dublin, Dublin 2, Ireland; 'Medical and Biological Sciences Building, University of St \\ Andrews, North Haugh, St Andrews, Fife, KY16 9TF.
}

\begin{abstract}
Cellular redox potential is important for the control and regulation of a vast number of processes occurring in cells. When the fine redox potential balance within cells is disturbed it can have serious consequences such as the initiation or progression of disease. It is thought that a redox gradient develops in cancer tumours where the peripheral regions are well oxygenated and internal regions, further from vascular blood supply, become starved of oxygen and hypoxic. This makes treatment of these areas more challenging as, for example, radiotherapy relies on the presence of oxygen. Currently techniques for quantitative analysis of redox gradients are limited. Surface enhanced Raman scattering (SERS) nanosensors (NS) have been used to detect redox potential in a quantitative manner in monolayer cultured cells with many advantages over other techniques. This technique has considerable potential for use in multicellular tumour spheroids (MTS) - a three dimensional (3D) cell model which better mimics the tumour environment and gradients that develop. MTS are a more realistic model of the in vivo cellular morphology and environment and are becoming an increasingly popular in vitro model, replacing traditional monolayer culture. Imaging techniques such as transmission electron microscopy (TEM), scanning electron microscopy (SEM) and helium ion microscopy (HIM) were used to investigate differences in morphology and NS uptake in monolayer culture compared to MTS. After confirming NS uptake, the first SERS measurements revealing quantitative information on redox potential in MTS were performed.
\end{abstract}

Keywords: MTS, SERS, TEM, HIM, NS, redox, cancer, tumour

\section{INTRODUCTION}

Cell culture is used extensively for the in vitro study of cellular characteristics including morphology, chemical composition and response to drug and therapy treatment. Typically cells are cultured as a monolayer growing on a flat surface such as a petri dish or flask. While this simple setup has been used to provide invaluable cellular information, it suffers from the drawback that the cells are not growing in a realistic physiological environment. In vivo cells exist in a 3D environment where their primary physical contacts are with surrounding cells and the extracellular matrix (ECM). ${ }^{1}$ In monolayer culture there is very little contact with other cells - the primary contacts being with the flat surface on which they are growing and the media they are growing in. The 3D environment in vivo gives rise to important differences in cellular characteristics compared to monolayer culture and for this reason 3D cell culture techniques are now being more frequently employed. ${ }^{2}$ In particular 3D techniques are useful in the study of cancer as, when a tumour grows, gradients in characteristics such as oxygen concentration, $\mathrm{pH}$ and nutrient concentration develop as the central regions of a tumour mass become further from the well organised vasculature of the body providing oxygen and nutrients to cells.,

Multicellular tumour spheroids (MTS) are a 3D cell culture model for the study of cancer. ${ }^{1,5}$ Due to the 3D nature of this model, techniques for analysis of cells grown in this way are complicated particularly by instrumental limitations in $\mathrm{z}$ imaging depths. ${ }^{6,7}$ Traditional histology techniques have been used to analyse paraffin embedded and sectioned MTS for attributes such as growth characteristics ${ }^{8}$; changes in morphology; hypoxia using reporters such as Hypoxyprobe ${ }^{\mathrm{TM} 9}$; and protein expression using immunohistochemistry ${ }^{10}$. These techniques have 
limitations in that they cannot be performed on live cells and fixing, embedding and sectioning can introduce artefacts and deformities. Fluorescence ${ }^{11}$ and bioluminescence ${ }^{12}$ have also been used when analysing characteristics such as $\mathrm{pH}$ and nutrient concentration but imaging depth is still a limitation. Other techniques such as transmission electron microscopy (TEM) $)^{13,14}$ and scanning electron microscopy (SEM) ${ }^{15-17}$ have been used to analyse morphological characteristics of MTS but again require fixing and, in the case of TEM, sectioning of samples.

A characteristic of particular interest in cancer tumours is intracellular redox potential. This is a measure of the oxidising potential of the intracellular environment and exists as a delicate balance between oxidants such as reactive oxygen species (ROS), antioxidant enzymes and reductants such as NADH/NADPH. ${ }^{18-22}$ Intracellular redox potential is tightly regulated in healthy cells and when this is disrupted disease can often progress. ${ }^{23-25}$ Disease is often associated with excessive ROS and oxidative stress at one end of the scale and lack of oxygen and hypoxia at the other. This is an important characteristic to consider in cancer tumours as cells near the centre of a tumour mass become increasingly far from the organised blood supply carrying oxygen to cells. Currently techniques for measuring hypoxia in MTS as a tumour model are limited and the 'gold standard' technique is to use small molecules like Hypoxyprobe ${ }^{\mathrm{TM} 26-29}$ which are reductively metabolized under hypoxic conditions and detected in paraffin embedded and sectioned samples by antibody detection and either colorometric or fluorescence detection. These probes give limited quantitative information however as they only report on whether the environment was above or below a certain limit. They also require fixing and sectioning MTS and therefore cannot be used for live cell dynamic measurements.

Our group has previously used SERS NS to monitor intracellular redox potential quantitatively in live cells in monolayer culture. ${ }^{30-32}$ In this technique, NS are prepared by attaching redox sensitive probe molecules to gold nanoparticles (NPs) and delivered to cells, SERS spectra are then collected and allow redox potential to be determined by ratiometric analysis of resultant signals. In this paper we extend this technique to MTS to allow quantitative analysis of redox potential in 3D culture and combine it with imaging techniques for analysis of morphological differences between monolayer and MTS culture and NS uptake. Specifically, we used TEM to analyse NS uptake and probe morphological differences between monolayer culture and MTS; Helium Ion Microscopy (HIM) $)^{33}$ (a relatively new technique that provides increased image resolution in comparison to SEM and removes the need for sample coating) to reveal morphological differences of MTS compared to monolayer culture and provide information on NS uptake at a surface level and SEM to reveal NS uptake below the surface of MTS.

\section{METHODOLOGY}

\section{NS functionalisation}

Aqueous suspensions of NPs $\left(2.2 \times 10^{9}\right.$ particles $\left./ \mathrm{ml}, 3.65 \mathrm{pM}\right)$ were purchased from Nanospectra Biosciences. NPs had a $150 \mathrm{~nm}$ diameter with a $125 \mathrm{~nm}$ silica core and $25 \mathrm{~nm}$ gold shell and were resonant at $782 \mathrm{~nm}$. For NQ-NP (where NQ refers to a naphthaquinone reporter), NPs were functionalised with $100 \mu \mathrm{M}$ NQ solution overnight in $10 \%$ EtOH solution. Functionalised NQ-NPs were washed 3 times with water. For AQ-NP (where AQ refers to an anthraquinone reporter), NPs were functionalised with $100 \mu \mathrm{M}$ AQ solution overnight in $1 \%$ DMSO solution. Functionalised AQ-NPs were washed 2 times with water.

\section{General Cell Culture Procedures}

All cellular work was carried out using MCF7 human breast cancer cells. Cells were cultured in Dulbecco's Modified Eagle's Medium (DMEM; Gibco; 31885-023), supplemented with penicillin/streptomycin (Invitrogen, 10,000 units $/ \mathrm{ml}$ ) and $10 \%$ heat-inactivated Foetal Calf Serum (FCS, Sigma). Cells were incubated at $37{ }^{\circ} \mathrm{C}$ and $5 \% \mathrm{CO}_{2}$ in a humidified incubator.

For monolayer culture $1 \times 10^{5}$ cells were plated on a coverslip in a 6 well plate or $35 \mathrm{~mm}$ glass bottomed imaging dish. The following day, growth media was replaced with media without FCS or remained the same. After 1-2 hrs, functionalised NS were added at a concentration of approximately $170 \mathrm{fM}$ and cells were incubated 
overnight. The next day, media was removed, cells were washed and media was replaced with normal growth media before performing SERS measurements.

For MTS, cells were split into $25 \mathrm{~cm}^{3}$ flasks. The following day growth media was replaced with media without FCS. After 1-2 hrs, functionalised NS were added at a concentration of approximately $200 \mathrm{fM}$ and cells were incubated overnight. The next day cells were trypsinised and resuspended in growth media before forming $20 \mu 1$ hanging drops of cell suspension on the lid of a petri dish. Growth media was added to the petri dish and the lid placed back on and MTS were grown for 6 days.

\section{General SERS information}

SERS spectra were recorded with a Reinshaw inVia Raman Microscope and Spectrometer. A $785 \mathrm{~nm}$ diode laser with $\sim 6 \mathrm{~mW}$ power at the sample was used, along with a $10 \times$ and $50 \times$ Olympus objective to focus laser light onto the gold NS. SERS spectra were processed using WIRE ${ }^{\mathrm{TM}} 2.0$ software and Origin 8.5.

\section{Intracellular SERS measurements}

When performing SERS measurements in cells a line focus laser was used. A rough map was firstly performed over a cell with an X step of $5.0 \mu \mathrm{m}$ and a Y step of $3.56 \mu \mathrm{M}, 1580 \mathrm{~cm}^{-1}$ center and 2 or $3 \mathrm{~s}$ acquisition time at $50 \%$ laser power. Where a signal was observed in the resulting spectra, coordinates were noted and a more detailed map with an X step of $1 \mu \mathrm{m}$ and a Y step of $1.19 \mu \mathrm{m}, 1580 \mathrm{~cm}^{-1}$ center and $30 \mathrm{~s}$ acquisition time at $10 \%$ laser power was recorded. This generated 147 spectra in just under 4 minutes.

\section{SERS data processing}

Spectra were analysed using Origin 8.5 software. Firstly all spectra from a single map were analysed by eye to determine the spectra with NQ-NP signals present. For all spectra with signals smoothing was performed 4 times using a Savitzky-Golay algorithm and 9 points of window. An 8 point baseline was then subtracted by manually placing the points. Peak intensities of peaks in the region of $1570-1588 \mathrm{~cm}^{-1}$ and $1630-1648 \mathrm{~cm}^{-1}$ were then determined using the peak finding function and the $1^{\text {st }}$ derivative method. The ratio of the peak intensity at $1630-$ $1648 \mathrm{~cm}^{-1}$ to that at $1570-1588 \mathrm{~cm}^{-1}$ was used along with calibration data from previous work in our group ${ }^{30}$ to determine redox potential from each spectrum.

\section{Transmission electron microscopy (TEM)}

Cells or cell spheroids were fixed in $0.5 \%$ gluteraldehyde in $0.1 \mathrm{M}$ sodium cacodylate or PBS for about $2 \mathrm{hrs}$. Cells in monolayer culture were scraped and transferred to an eppendorf tube where they were pelleted by centrifugation before removing fixative and adding PBS. Cell spheroids were fixed in an eppendorf tube before removing excess fixative and adding PBS.

Osmium tetroxide solution was prepared by mixing 1 part $4 \%$ osmium tetroxide, 1 part $6 \%$ potassium ferrocyanide, 1 part $0.4 \mathrm{M}$ sodium cacodylate and 1 part $\mathrm{dH}_{2} \mathrm{O}$. Excess PBS supernatant was removed from the samples and osmium tetroxide solution $(100-200 \mu \mathrm{l}$ per sample) was added and cells incubated for 10-15 minutes. Osmium tetroxide solution was removed and the samples were washed with distilled water $(3 \times 5 \mathrm{~min})$. Using a glass vial rotator for subsequent steps, $50 \%$ ethanol was added to the samples and they were transferred into small glass vials. After 10 mins, $50 \%$ ethanol was removed and $70 \%$ ethanol added to each sample, making sure the sample did not dry out during exchange. A further series of graded ethanol steps were carried out $(80 \%$, $90 \%, 95 \%$ and $2 \times 100 \%$ ) with 10 min incubation per step. Following this $2 \times 15$ min propylenoxide incubations were carried out, before adding a 1:1 mix of epoxy resin in propylenoxide and incubating overnight with a sealed lid. The next morning, lids were removed from the vials and the samples were incubated for a further 3 hours on the rotator until the propylenoxide had evaporated. Cell fragments were taken out, gently blotted on filter paper, placed inside the PVC lids and fresh epoxy resin added. After a further 4 hours incubation, the blotting of fragments was repeated and the fragments were placed in PVC capsules for making the final blocks and filled with fresh epoxy resin. Capsules were placed in oven at $60^{\circ}$ for 24 hours. 
$70 \mathrm{~nm}$ sections were taken using a diamond knife. Sections were positioned on copper grids and stained with lead citrate before examining and photographing with a Jeol JEM transmission electron microscope connected to a digital camera.

\section{Helium ion microscopy (HIM)}

Monolayer cultured cells grown on a coverslip and incubated overnight with functionalised NPs as described previously or MTS grown as described previously were fixed in $0.5 \%$ gluteraldehyde in $0.1 \mathrm{M}$ sodium cacodylate or PBS. Samples were mounted on a sticky carbon stub and analysed using a Zeiss Orion Plus Helium Ion Microscope running at $30 \mathrm{kV}$ with $10 \mu \mathrm{m}$ aperture, an Everhart Thornley detector and charge neutralization achieved using an electron flood gun.

\section{Scanning electron microscopy (SEM)}

Samples were prepared as for SEM and analysed using a Zeiss Ultra SEM.

\section{RESULTS}

Previously SERS NS functionalised with redox active molecules NQ and AQ have been used to measure intracellular redox potential in NIH/3T3 mouse fibroblast ${ }^{30}, \mathrm{~A} 549^{31}$ and EA.hy $926^{32}$ cells in monolayer culture. The primary goal of this research was to use these NS to measure intracellular redox potential in MTS, in particular MCF7 breast cancer MTS.

\section{TEM}

TEM was used primarily to confirm NS uptake but could also be used to reveal morphological differences over a restricted field of view. Figure 1 compares monolayer and MTS cell morphology by TEM where the primary difference is the increase in cell-cell interactions in MTS, mimicking better the in vivo environment.

TEM was used to confirm uptake by MCF7 cells in a similar way to previous work and secondly to confirm that NS remained in cells over extended periods of time up to 7 days after incubation with cells. Previous work with monolayer cells employed an overnight incubation with NS before taking SERS measurements the following day but as MTS take several days to grow, we used TEM to confirm that NS were maintained inside cells over a longer time period. To form MTS that contained NS, NS were added in monolayer culture before forming MTS from the resultant cell suspension and growing for 6 days in hanging drops - as a comparison between 2D and 3D we also examined cells that were incubated with NS overnight before growing for either a further 6 days in monolayer culture or as MTS.

Figure 2 shows TEM images of NQ-NPs in MCF7 cells in monolayer culture after 24 hours incubation with NQNPs before fixing. NQ-NPs were found both free in the cytosol (Figure 2(b)) and inside endosome structures (Figure 2(c)). This confirmed uptake of NS to MCF7 cells. Interestingly some NQ-NPs were found on the outside edge of cells (Figure 2(a)), as if in the process of being taken up.
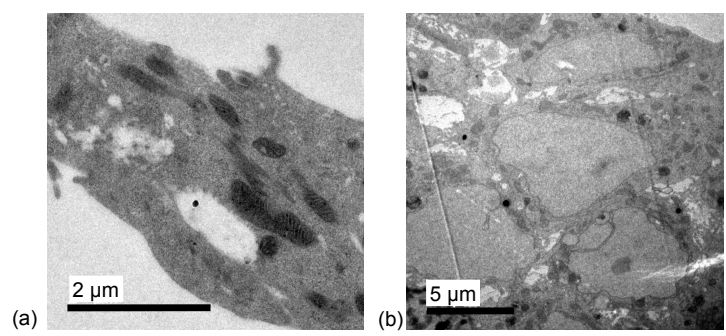

Figure 1 shows TEM images of MCF7 cells in monolayer culture (a) and MTS (b) where differences in cell morphology can be visualised. 
TEM images from MCF7 cells which were incubated for 24 hours with NQ-NPs in monolayer culture before a further 6 days growth in 2D (Figure 3) and as MTS (Figure 4) revealed that NQ-NPs remained in the cells for this extended period of time. Again NQ-NPs were found both in the cytosol and endosome structures for cells grown over extended periods of time in monolayer and MTS.

(a)

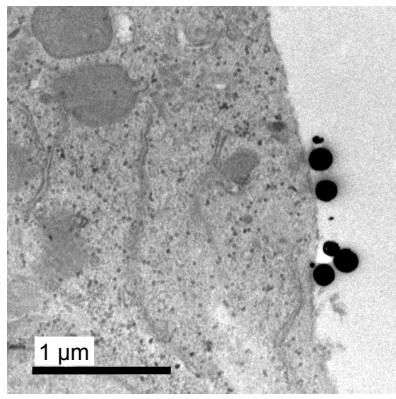

(b)

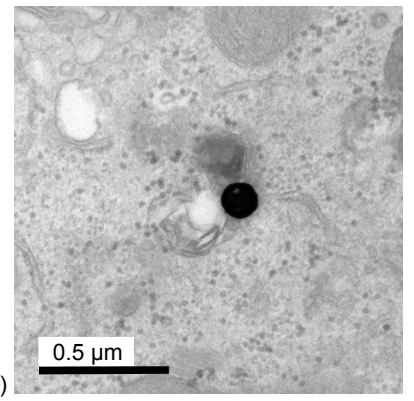

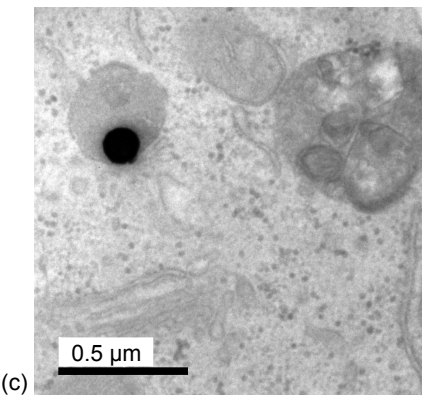

(c)

Figure 2 (a)-(c) illustrate TEM images obtained for MCF7 cells grown in monolayer culture and incubated with NQNPs for 24 hours before fixing.

(a)

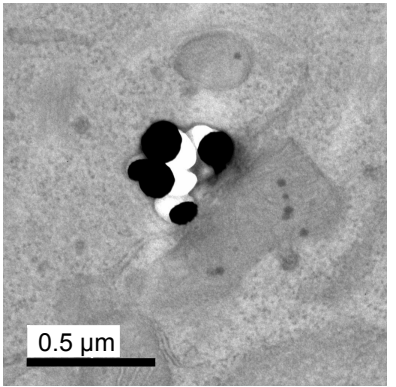

(b)

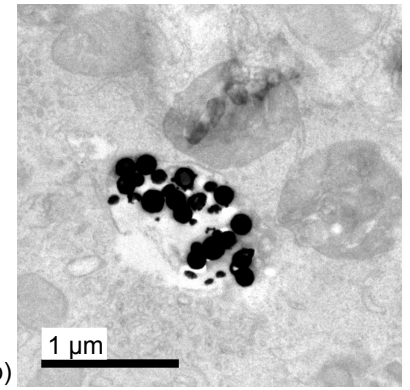

(c)

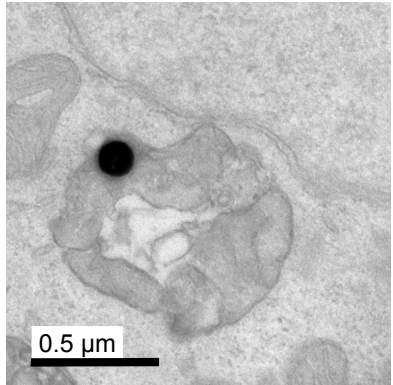

Figure 3 (a)-(c) illustrate TEM images obtained for MCF7 cells grown in monolayer culture and incubated with NQNPs for 24 hours followed by a further 6 days growth in monolayer culture before fixing.

(a)

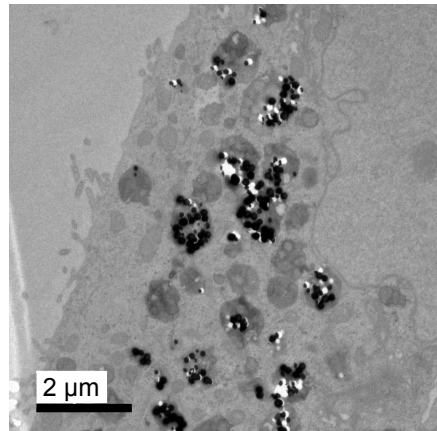

(c)

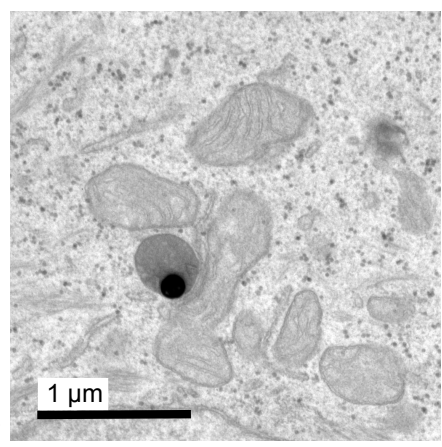

(b)

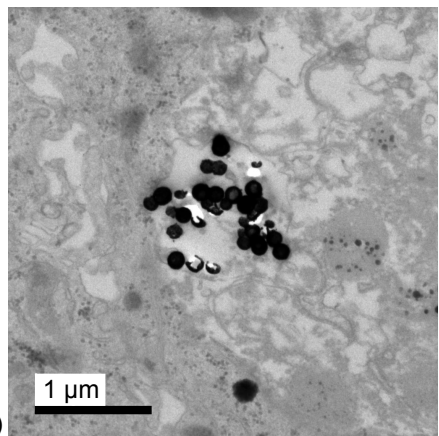

(d)

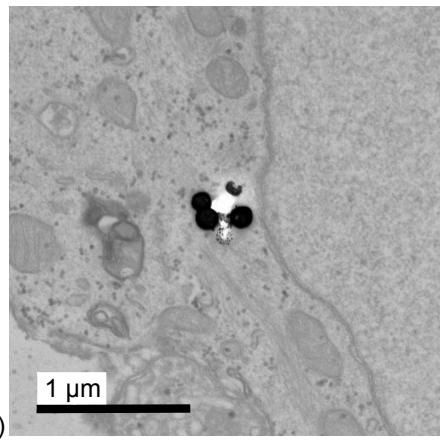

Figure 4 (a)-(d) illustrate TEM images obtained for MCF7 cells grown in monolayer culture and incubated with NQNPs for 24 hours followed by a further 6 days growth as MTS before fixing. 


\section{SEM \& HIM}

HIM provides increased resolution to SEM for comparison of morphological features of MTS and monolayer cultured cells. ${ }^{33}$ While SEM often requires samples to be coated in gold prior to analysis, this is not required for HIM. As coating can remove a lot of surface features HIM allows surface detail to be more accurately visualised in comparison to SEM. Figure 5 compares cell morphology of monolayer cultured MCF7 cells (Figure 5(a)) to MTS over both a larger field of view to image the whole MTS (Figure 5(b)) and a smaller field of view showing cell-cell interactions (Figure 5(c)).

It is possible to use HIM to visualise the interaction of NS with cells. Figure 6 shows NS interacting with cells in monolayer culture. This is limited to visualizing NS on the surface of cells as this technique is highly surface sensitive. Interestingly NS with different functionalities appeared to interact differently with cells. Figure 6(a) and (b) show the interaction of AQ-NPs with cells and Figure 6(c) and (d) show the interaction of NQ-NPs with cells. AQ-NPs appear to be more aggregated and it is possible that cells are either releasing clumps of NS that have been coated in protein within the cell, or depositing extra cellular matrix onto NS outside the cell - Figure 6(b) shows some extracellular material encapsulating AQ-NPs. Aggregates of NQ-NPs are also visible as in Figure 6(d) however they are fewer and often only single NQ-NPs are seen as in Figure 6(c). This is likely due to the differing properties of $\mathrm{NQ}$ and $\mathrm{AQ}-\mathrm{AQ}$ is more hydrophobic.

While HIM provided some information regarding NS interaction with the surface of cells in monolayer culture it was difficult to extend this technique into MTS as there were fewer instances of NS being present on the surface of cells on the outside of MTS. In order to try and visualise NS in MTS, SEM was performed using backscattered electron detection to detect NS just below the surface of cells (Figure 7). This technique allowed NS to be visualised successfully however it was only possible to see NS just below the surface of cells so had limited applicability for looking at distribution over the whole MTS. These images also allowed comparison of resolution to those captured using HIM where much greater resolution was achieved in HIM.

(a)
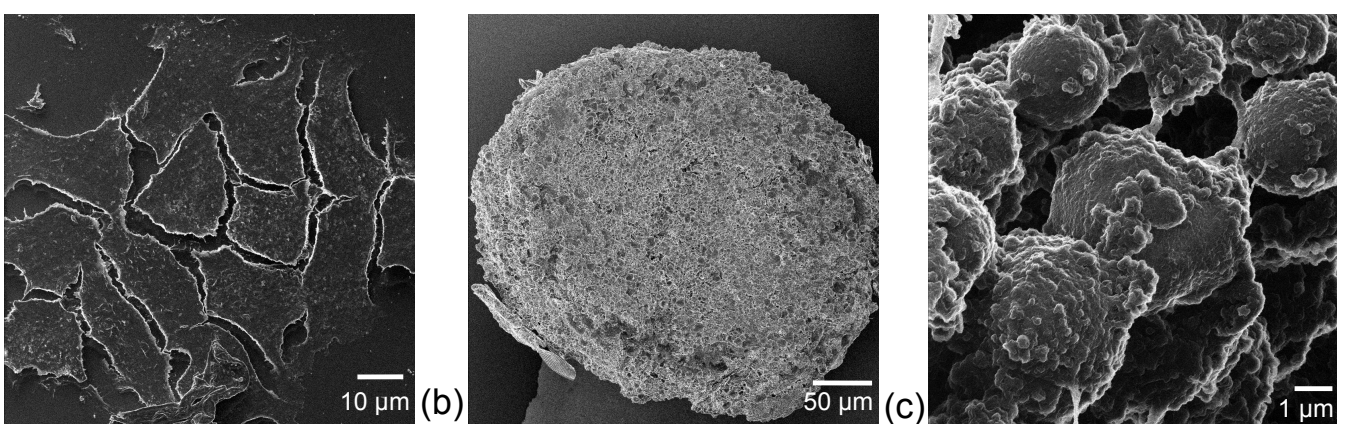

Figure 5 shows HIM images of MCF7 cells growing in monolayer culture (a) and as MTS with a larger field of view to image a whole MTS (b) and smaller field of view to image more detailed cell-cell interactions in MTS (c).

(a)
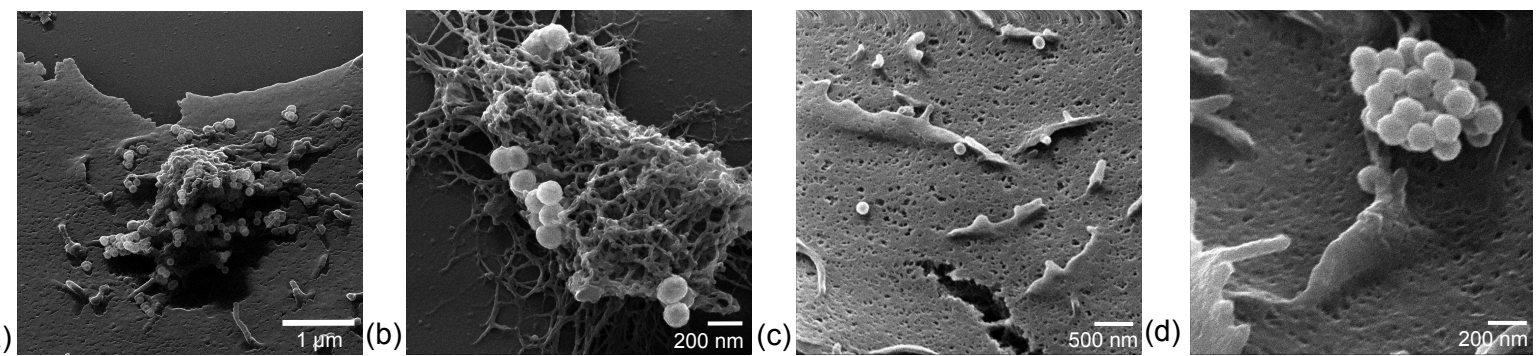

Figure 6 shows HIM images of AQ-NPs interacting with the surface of MCF7 cells grown in monolayer culture (a), as well extracellular material (b) and NQ-NPs interacting with the surface of MCF7 cells (c and d). 
(a)

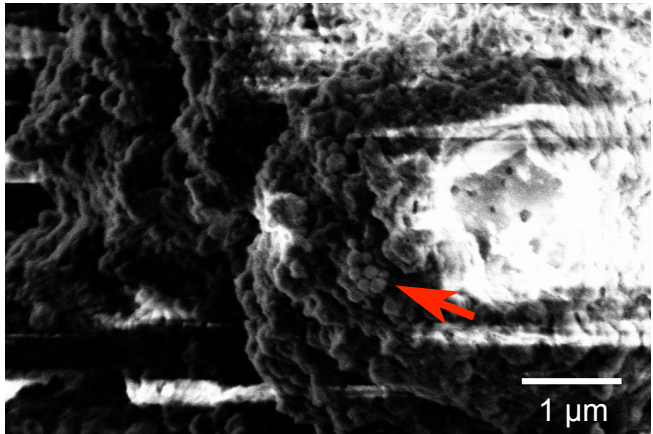

(b)

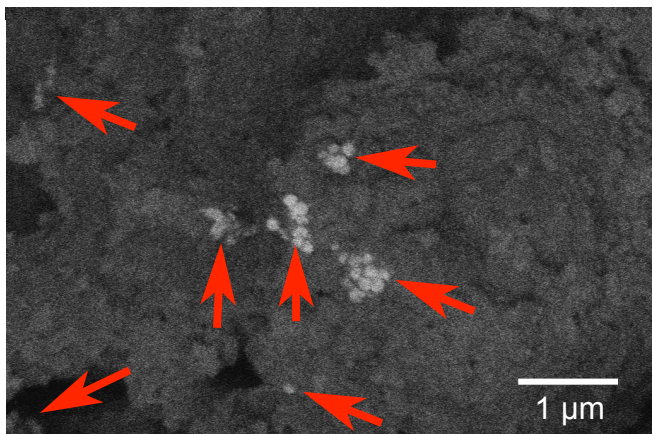

(c)

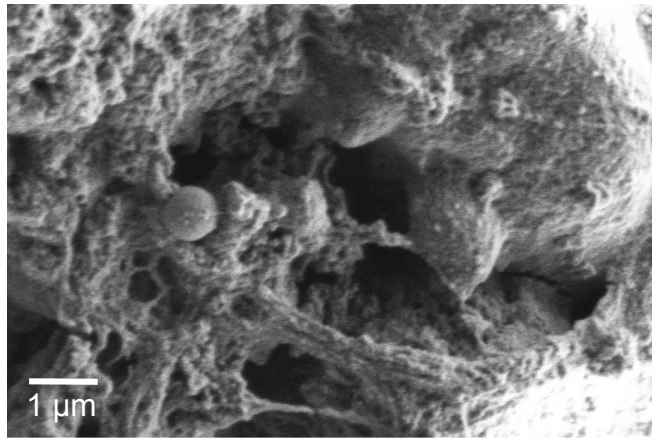

(d)

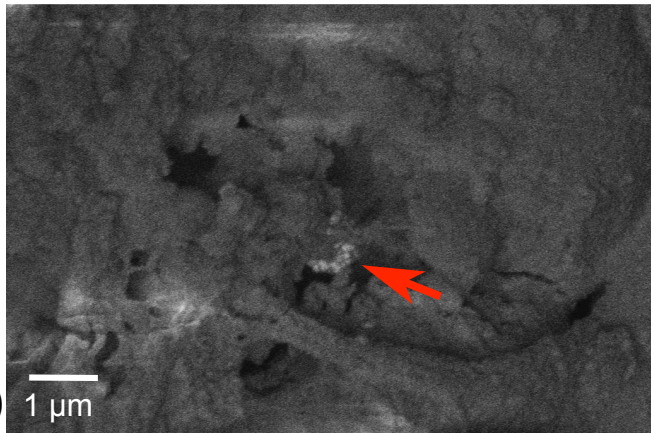

Figure 7 shows SEM images of regions of MCF7 cell MTS where the same area was imaged with secondary (a and c) and backscattered (b and d respectively) electrons. Red arrows point to areas where NS are visible.

\section{SERS}

After confirming NS uptake in both monolayer culture and MTS, SERS was used to investigate redox potential in both cases using the probe NQ-NP (Figure $8(\mathrm{a})$ ). ${ }^{30}$ For calibration, spectra of NQ-NPs dried onto a slide and covered by buffer were recorded at different redox potential values set using a potentiostat (as previously published). The ratiometric response of signals in the spectrum assigned to $\mathrm{C}=\mathrm{O}$ stretching at $1630-1648 \mathrm{~cm}^{-1}$ and aromatic ring breathing at $1577-1600 \mathrm{~cm}^{-1}$ was used to construct a calibration plot and equation for determining redox potential. The $\mathrm{C}=\mathrm{O}$ stretching signal increases in intensity with increasing oxidising potential of the environment due to an increased proportion of $\mathrm{NQ}$ being present in the oxidised form where $\mathrm{C}=\mathrm{O}$ bonds are present.

For monolayer culture MCF7 cells were plated and incubated with NQ-NPs overnight as described in the experimental section before carrying out SERS on the cells. Figure 8(b) shows a typical SER spectrum of NQNPs from a cell in monolayer culture, which gave a redox potential of $-260 \mathrm{mV}$. Figure 9 summarises redox potential obtained in monolayer culture cells giving a mean potential of $-260 \pm 4.5 \mathrm{mV}$.

After applying the previously established technique to the MCF7 cells of choice in monolayer culture, resulting in redox potential values expected, work began to expand this technique to samples prepared in 3D as MTS. As already discussed and detailed in the experimental section, cells were incubated with NQ-NPs in monolayer culture before growing the resultant cell suspension in hanging drops to form MTS. Regions of MTS were then mapped in a similar way to monolayer cultured cells as detailed in the experimental section. As MTS are much larger, this meant only small regions of MTS could be mapped at a time and at this stage, detection of whole MTS variations in redox potential were not possible. The number of spectra obtained for a single map was much greater than that for monolayer cultured cells, possibly due to the additional z direction from which signals could be obtained. Figure 9 illustrates the distribution of redox potential values obtained from a single map of NQ-NPs from an MTS compared to the previously obtained values for monolayer culture. The average redox potential of $-268 \pm 5.9 \mathrm{mV}$ was more negative than that for the values obtained in monolayer culture, which would be expected from the more hypoxic nature of MTS. Some spectra had no $\mathrm{C}=\mathrm{O}$ stretching peak (fully reduced NQ). A one way ANOVA confirmed that redox potential values in monolayer culture compared to MTS were 
significantly different at the 0.05 level. Not only was mean redox potential different between monolayer culture and MTS but MTS showed a higher standard deviation and more widespread redox potential values. This was expected as a gradient develops in MTS so a wider range of redox values would be expected across this gradient.

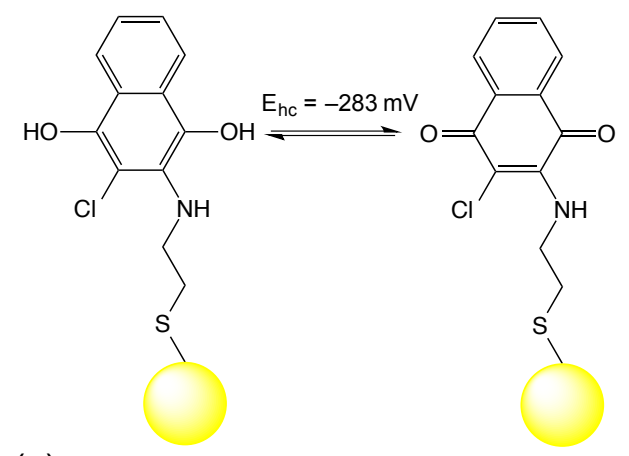

(a) (b)

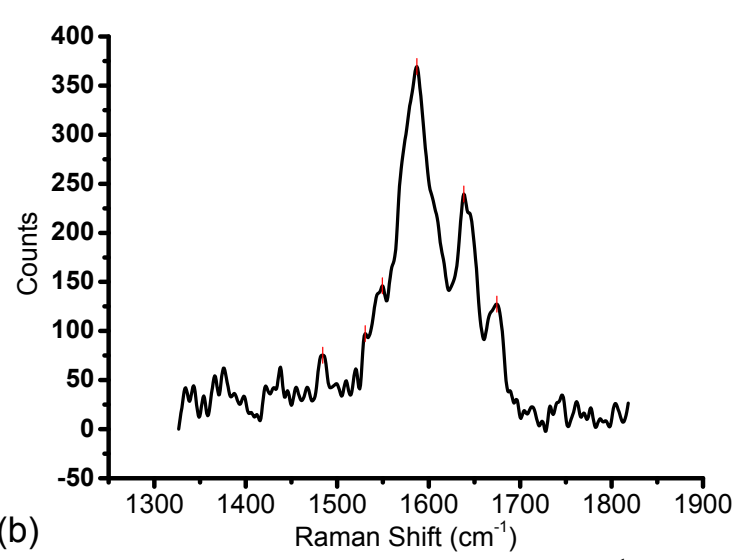

Figure 8 shows a typical SERS spectrum obtained from NQ-NPs in MCF7 cells with peaks at $1638 \mathrm{~cm}^{-1}$ and 1587 $\mathrm{cm}^{-1}$ assigned to $\mathrm{C}=\mathrm{O}$ stretch and ring breathing respectively.

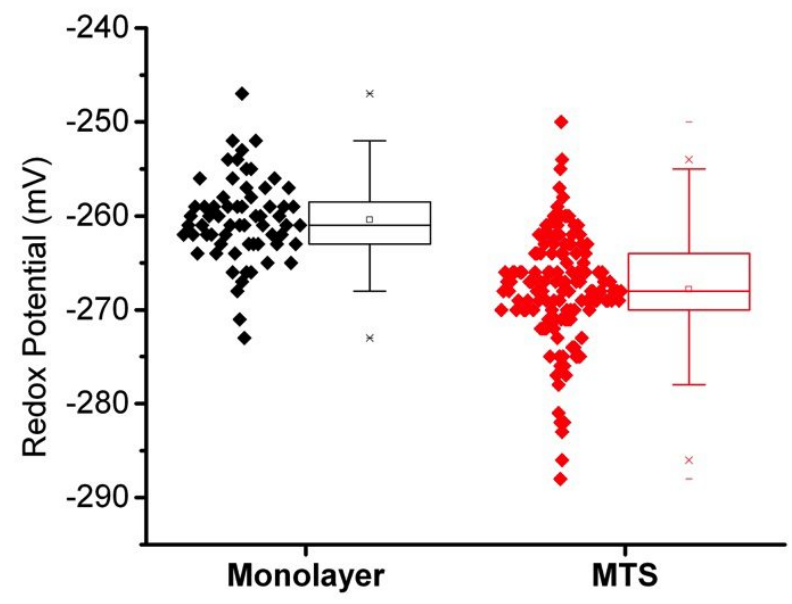

Figure 9 illustrates the distribution of redox potential values obtained using SERS of NQ-NPs in MCF7 cells in monolayer culture and grown as MTS. A one way ANOVA confirmed that the two sets of data were significantly different at the 0.05 level.

\section{CONCLUSIONS}

MTS are becoming increasingly important as a cell culture model that mimics the in vivo environment better than the traditional monolayer culture and these results reveal some of the important characteristics that develop in this 3D environment. Cell morphology and NS uptake were measured and monitored using TEM, SEM and HIM. TEM was used primarily to confirm both the uptake of functionalised NPs to MCF7 cells in monolayer culture for the first time and that they remained present in cells in both monolayer culture and MTS over extended periods of time up to one week. HIM is a relatively new technique that was used for the first time to study NS uptake in MCF7 cells in monolayer and MTS culture and reveal significant morphological differences between cells in monolayer culture and MTS. While it provided little information about NS uptake in MTS, detection of backscattered electrons by SEM allowed some NS to be visualised in MTS but also revealed the much greater image resolution of HIM compared to SEM. SERS has been used for the first time to reveal quantitative 
information regarding intracellular redox potential in MCF7 MTS. These initial experiments used the redox probe NQ-NP, previously developed in our group, to probe intracellular redox potential in MCF7 cells in both monolayer culture and MTS. As expected, results revealed a significantly lower and more varied intracellular redox potential state in MTS compared to monolayer culture. All techniques collectively revealed increased information on NS uptake in monolayer culture and MTS, important differences in morphology between monolayer culture and MTS and important quantitative differences in characteristics of MTS, specifically intracellular redox potential, in comparison to monolayer culture.

\section{ACKNOWLEDGEMENTS}

The authors gratefully acknowledge the School of Chemistry at the University of Edinburgh and The School of Medicine at the University of St Andrews. This research received support from the QNano Project http://www.qnano-ri.eu which is financed by the European Community Research Infrastructures under the FP7 Capacities Programme (Grant No. INFRA-2010-262163), and its partner Trinity College Dublin. The authors thank Dr John Lucocq and Dr Christian Hacker at the University of St Andrews for TEM work.

\section{REFERENCES}

[1] Page, H., Flood P. and Reynaud, E. G., " Three-dimensional tissue cultures: current trends and beyond", Cell Tissue Res., 352, 123-131 (2013).

[2] Hirschhaeuser, F., Menne, H., Dittfeld, C., West, J., Mueller-Klieser, W. and Kunz-Schughart, L.A. " Multicellular tumor spheroids: An underestimated tool is catching up again", J. Biotechnol., 148, 3-15 (2010).

[3] Hanahan, D. and Weinberg, R.A., " Hallmarks of Cancer: The Next Generation", Cell, 144, 646-674 (2011).

[4] Vaupel, P. " Tumor Microenvironmental Physiology and Its Implications for Radiation Oncology", Semin. Radiat. Oncol., 14, 198-206 (2004).

[5] Lin, R.-Z. and Chang, H.-Y, " Recent advances in three-dimensional multicellular spheroid culture for biomedical research", Biotechnol. J., 3, 1172-1184 (2008).

[6] Jamieson, L. E., Harrison, D.J. and Campbell, C. J. "Chemical Analysis of Multicellular Tumour Spheroids", Analyst (2015) (accepted).

[7] Pampaloni, F., Ansari, N., and Stelzer, E. H. K, " High-resolution deep imaging of live cellular spheroids with light-sheet-based fluorescence microscopy", Cell Tissue Res., 352, 161-177 (2013).

[8] K. Groebe, 1991, 799, 792-799.

[9] Anada, T., Fukuda, J., Sai, Y. and Suzuki, O., " An oxygen-permeable spheroid culture system for the prevention of central hypoxia and necrosis of spheroids", Biomaterials, 33, 8430-8441 (2012).

[10] Lee, J. M., Mhawech-Fauceglia, P., Lee, N., Parsanian, L. C., Lin, Y. G., Gayther, S. A. and Lawrenson, K., " A three-dimensional microenvironment alters protein expression and chemosensitivity of epithelial ovarian cancer cells in vitro", Lab. Invest., 93, 528-542 (2013).

[11] Rotin, D., Steele-Norwood, D., Grinstein, S. and Tannock, I., " Requirement of the $\mathrm{Na}^{+} / \mathrm{H}^{+}$Exchanger for Tumor Growth", 49, 205-211 (1989).

[12] Walenta, S., Doetsch, J., Mueller-Klieser, W. and Kunz-Schughart, L. A., " Metabolic Imaging in Multicellular Spheroids of Oncogene-transfected Fibroblasts", J. Histochem. Cytochem., 48(4), 509-522 (2000).

[13] Sutherland, R. M., McCredie, J. A. and Inch, W. R., "Growth of Multicell Spheroids in Tissue Culture as a Model of Nodular Carcinomas", J. Natl. Cancer Inst., 46, 113-120 (1971).

[14] Nederman, T., Norling, B., Glimelius, B., Carlsson, J. and Brunk, U., "Demonstration of an Extracellular Matrix in Multicellular Tumor Spheroids", 44(7), 3090-3097 (1984).

[15] Ho, W. Y., Yeap, S. K., Ho, C. L., Rahim, R. A. and Alitheen, N.B., "Development of Multicellular Tumor Spheroid (MCTS) Culture from Breast Cancer Cell and a High Throughput Screening Method Using the MTT Assay", PLoS One, 7(9), e44640 (2012). 
[16] Santini, M. T., Rainaldi, G. and Indovina, P. L., "Apoptosis, cell adhesion and the extracellular matrix in the three-dimensional growth of multicellular tumor spheroids", Crit. Rev. Oncol. Hematol., 36, 75-87 (2000).

[17] Ma, H., Jiang, Q., Han, S., Wu, Y., Tomshine, J. C., Wang, D., Gan, Y., Zou, G. and Liang, X.-J., "Multicellular Tumor Spheroids as an In Vivo-Like Tumor Model for Three-Dimensional Imaging of Chemotherapeutic and Nano Material Cellular Penetration", Mol. Imaging, 11(6), 487-498 (2012).

[18] Kemp, M., Go, Y.-M. and Jones, D. P., " Nonequilibrium thermodynamics of thiol/disulfide redox systems: A perspective on redox systems biology", Free Radic. Biol. Med., 44, 921-937 (2008).

[19] Zhang, Y., Hong, H. and Cai, W., " Imaging with Raman Spectroscopy", Curr. Pharm. Biotechnol., 11(6), 654-661 (2011).

[20] Schafer, F. Q. and Buettner, G. R., "Redox Environment of the Cell as Viewed Through the Redox State of the Glutathione Disulfide/Glutathione Couple", Free Radic. Biol. Med., 30(11), 1191-1212 (2001).

[21] Filomeni, G., Rotilio, G. and Ciriolo, M. R., " Disulfide relays and phosphorylative cascades: partners in redox-mediated signaling pathways", Cell Death Differ., 12, 1555-1563 (2005).

[22] Mallikarjun, V., Clarke, D. K. and Campbell, C. J., " Cellular redox potential and the biomolecular electrochemical series: A systems hypothesis", Free Radic. Biol. Med., 53, 280-288 (2012).

[23] Waris, G. and Ahsan, H., " Reactive oxygen species: role in the development of cancer and various chronic conditions", J. Carcinog., 5(14), (2006).

[24] Go, Y.-M. and Jones, D. P., " Cysteine/cystine redox signaling in cardiovascular disease", Free Radic. Biol. Med., 50, 495-509 (2011).

[25] Dewhirst, M. W., Cao, Y. and Moeller, B., " Cycling hypoxia and free radicals regulate angiogenesis and radiotherapy response", Nat. Rev. Cancer, 8, 425-437 (2008).

[26] Chemicon International, "Hypoxyprobe ${ }^{\mathrm{TM}}-1$ Plus Kit for the Detection of Tissue Hypoxia", accessed March

2015 , http://webcache.googleusercontent.com/search?q=cache:8bxU7yy_OUoJ:www.ibrarian.net/navon/paper/ HypoxyprobeTM_1_Plus_Kit_for_the_Detection_of_Tis.pdf\%3Fpaperid\%3D4633961+\&cd=5\&hl=en\& $\mathrm{ct}=\mathrm{clnk} \& \mathrm{gl}=\mathrm{uk}$.

[27] Raleigh, J. A., Calkins-Adams, D. P., Rinker, L. H., Ballenger, C. A., Weissler, M. C., Fowler, W. C., Novotny, D. B. and Varia, M. A., " Hypoxia and Vascular Endothelial Growth Factor Expression in Human Squamous Cell Carcinomas Using Pimonidazole as a Hypoxia Marker", Cancer Res., 58, 3765 3768 (1998).

[28] Varghese, A. J., Gulyas, S. and Mohindra, J. K., " Hypoxia-dependent Reduction of 1-(2-Nitro-1imidazolyl)-3-methoxy-2-propanol by Chinese Hamster Ovary Cells and KHT Tumor Cells in Vitro and in Vivo", 36, 3761-3765 (1976).

[29] Raleigh, J. A., Miller, G. G., Franko, A. J., Koch, C. J., Fuciarelli, A. F. and Kelly, D. A., " Fluorescence immunohistochemical detection of hypoxic cells in spheroids and tumours", Br. J. Cancer, 56, 395-400 (1987).

[30] Auchinvole, C. A. R., Richardson, P., McGuinnes, C., Mallikarjun, V., Donaldson, K., McNab, H. and Campbell, C. J., " Monitoring Intracellular Redox Potential Changes Using SERS Nanosensors", ACS Nano, 6(1), 888-896 (2012).

[31] Jiang, J., Auchinvole, C., Fisher, K. and Campbell, C. J., " Quantitative measurement of redox potential in hypoxic cells using SERS nanosensors", Nanoscale, 6, 12104-12110 (2014).

[32] Jamieson, L. E., Jaworska, A., Jiang, J., Baranska, M., Harrison, D. J. and Campbell, C. J., " Simultaneous intracellular redox potential and $\mathrm{pH}$ measurements in live cells using SERS nanosensors", Analyst, 140(7), 2330-2335 (2015).

[33] Hlawacek, G., Veligura, V., van Gastel, R. and Poelsema, B., " Helium ion microscopy", J. Vac. Sci. Technol. B Microelectron. Nanom. Struct., 32(2), 020801 (2014). 